\title{
Language Rehabilitation of Traumatic Brain Injury Patient by LORETA Z-Score Neurofeedback: A Single-Case Study
}

\author{
Farnaz Faridi1, Hayat Ameri ${ }^{*}$, Masoud Nosratabadi2, Seyed Majid Akhavan Hejazi3, and \\ Robert W. Thatcher 4 \\ ${ }^{1}$ Tarbiat Modarres University, Tehran, Iran \\ ${ }^{2}$ Department of Psychology, University of Social Welfare and Rehabilitation Sciences, Tehran, Iran \\ ${ }^{3} \mathrm{Head}$, Brain, and Spinal Cord Injuries Department, Rofeideh Hospital, University of Social Welfare and Rehabilitation \\ Sciences, Tehran, Iran \\ ${ }^{4}$ Applied Neuroscience, Inc., St. Petersburg, Florida, USA
}

\begin{abstract}
Traumatic brain injury (TBI) creates a variety of sequelae such as aphasia that can be highly challenging for clinicians when developing rehabilitation interventions. Therefore, the present study aimed to investigate the effectiveness of LORETA $z$-score neurofeedback (LZNFB) on language performance for a 21 -year-old male suffering from aphasia following TBI. To this end, LZNFB was applied while focusing on the language network for 15 sessions. The study used an experimental design with a pre-post comparison. Baseline and posttreatment comparisons were made on qEEG/LORETA metrics, aphasia symptoms, working memory, and attention. The results indicated clinical improvements in language, working memory, and attention performances after 15 sessions of LZNFB. Our findings suggest that LZNFB may have the potential to aid language performance among those with TBI.
\end{abstract}

Keywords: traumatic brain injury; LORETA neurofeedback; language; working memory; attention

Citation: Faridi, F., Ameri, H., Nosratabadi, M., Hejazi, S. M. A., \& Thatcher, R. W. (2021). Language rehabilitation of traumatic brain injury patient by LORETA $z$-score neurofeedback: A single-case study. NeuroRegulation, 8(2), 121-126. https://doi.org/10.15540/nr.8.2.121

*Address correspondence to: Hayat Ameri, Associate Professor of Linguistics, Tarbiat Modarres University, Tehran, Iran. Email: h.ameri@modares.ac.ir

Copyright: () 2021. Faridi et al. This is an Open Access article distributed under the terms of the Creative Commons Attribution License (CC-BY).
Edited by:

Rex L. Cannon, PhD, SPESA Research Institute, Knoxville, Tennessee, USA

Reviewed by:

Wesley D. Center, PhD, Liberty University, Lynchburg, Virginia, USA

Jon A. Frederick, PhD, Lamar University, Beaumont, Texas, USA

\section{Introduction}

Traumatic brain injury (TBI) is an injury to the brain and is typically caused by an acute injury to the head, neck, or face (Brown et al., 2019). The wide array of problems confronting those with TBI includes headache, fatigue, impaired memory, reduced attention, depression, aggression, anxiety, sleep disturbances, and sexual dysfunction (Barth et al., 1983). Several reports indicated that TBI can have lifelong impacts including changes in personality and behavior (Banks, 2007; Jackson et al., 2002).

The consequences of TBI are not limited to those changes but also lead to electroencephalographic (EEG) abnormalities, which can be focal or widespread (Brigo \& Mecarelli, 2019; Galovic et al.,
2017). Some studies demonstrated quantitative EEG (qEEG) changes in patients with TBI. For example, the attenuated alpha frequency in the posterior region and increased theta activity are the most common qEEG findings of individuals with TBI (Arciniegas, 2011; Lewine et al., 2019). Moeller et al. (2011) reported increased delta and theta bands and a decreased beta band in TBI due to the disruption of the cortical-thalamic network. Higher theta-alpha, theta-beta, and delta-alpha amplitude ratios and reduced EEG coherence were also noted in TBI (Modarres et al., 2017). Developing medical treatments that ameliorate the symptoms of TBI is of great importance, and neurofeedback (NF) is one such method.

A review of the literature shows promise for treating some symptoms of TBI with this modality (Gray, 2017). Ayers (1989) was the first to report positive 
effects of NF on TBI-related symptoms, finding improvements in a number of postconcussive symptoms experienced by patients, including decreased energy, depression, irritability, photophobia, attention deficit, dizziness, headache, and short-term memory loss. The role of NF in improving cognitive, behavioral, and physical dysfunctions among patients with $\mathrm{TBI}$ has been confirmed in previous studies (Bennett et al., 2018; Brown et al., 2019; Gray, 2017; Gupta et al., 2020; Hershaw et al., 2020; Kaser, 2020; Koberda, 2015a).

Although previous studies have shown that NF can mitigate many symptoms of $\mathrm{TBI}$, they have not specifically focused on language rehabilitation by NF. Nevertheless, language therapy produces clinically significant improvements in functional communication, better mood, and quality of life of people with TBI aphasia. Accordingly, the present study sought to evaluate the efficiency of LORETA $z$-score neurofeedback (LZNFB) to rehabilitate the language deficit in a patient with aphasia following TBI. LZNFB is one of the recent advanced technologies of NF that increases specificity by targeting brain network hubs (e.g., the language network) that are referred to as Brodmann areas. The advantage of using the $z$-score in LORETA NF is the ability to receive instant comparisons using a reference database of healthy individual $z$-scores (Thatcher, 2010). These instantaneous comparisons make it possible to find the link between patients' symptoms and the pertinent Brodmann areas (Thatcher, 2010).

In this study, it was hypothesized that LZNFB intervention could potentially enhance language performance in a patient with aphasia following TBI. To test this hypothesis at least in a single case investigation, 15 sessions LZNFB were applied to the language network.

\section{Methods}

\section{Case Description}

P.F. was a 21-year-old, right-handed male who suffered from aphasia after trauma. Ten months prior to our assessment, he had an accident, and his head had been hurt at the right inferior frontal area. After being unconscious for one month following the accident, the patient underwent surgery on his head. Table 1 presents the demographic information of the patient when he was hospitalized following the trauma. At the time of the assessment, he was alert and oriented and could follow commands, although his language performance was poor.

\begin{tabular}{|c|c|c|c|c|}
\hline \multicolumn{5}{|c|}{$\begin{array}{l}\text { Table } 1 \\
\text { Demographic Information of P.F When Hospitalized After } \\
\text { Trauma }\end{array}$} \\
\hline Severity & PTA & Age & LOC & GCS \\
\hline 7 & 277 & 21 & 30 & 6 \\
\hline
\end{tabular}

Note. The severity index is a number between 1 and 10 , indicating the severity of $\mathrm{TBI}$ based on discriminant classification. Values in the range of 1 to 3,3 to 5 , and $>5$ indicate mild, moderate, and severe head trauma, respectively. PTA: Posttraumatic amnesia; LOC: Loss of consciousness; GCS: Glasgow Coma Scale.

\section{Intervention}

Power spectral analyses were performed on 5-min segments of the eyes-closed resting state. An EEG was recorded from 19 scalp locations based on the international 10-20 system of electrode placement using the linked ear as a reference. Using a Medicom amplifier and EEG Studio Acquisition software, qEEG data were collected. In addition, editing and digital analysis of the qEEG data were conducted using NeuroGuide software and a comparative database. The protocol included LZNFB to focus on the language network in the symptom checklist, which was developed with the goal of linking symptoms to the areas of the brain. Brodmann areas (BA) in this language network include 22, 39, 40, 41, 42, 44, and 45. Learning reinforcement in neurofeedback was provided using television shows or animations that increased in size when meeting the difficulty thresholds.

The qEEG/LORETA analysis was completed by NEUROSTAT and NeuroGuide software. The available neurocognitive testing batteries (Persian aphasia battery, Stroop test, digit span, and word/nonword test) were used before and after LZNFB and compared using the Barlow formula. The formula for recovery percentage is as follows:

$$
\Delta A=\frac{A 2-A 1}{A 2}(100)
$$

As suggested by Barlow et al. (2007), if the results are greater than $15 \%$, we can conclude that the results are clinically significant and treatment is successful.

\section{Results}

The pretreatment qEEG demonstrated elevated levels of all brain waves except alpha in the frontal and temporal regions. After 20 LZNFB sessions, brain wave amplitudes were closer to values from 
the database, as reflected by reduced $z$-scores (Figure 1).

The percentage difference between the baseline and last session of treatment was computed, revealing that the largest changes were found in delta waves at $\mathrm{F} 7$ and in high beta waves at F8, T4, T5, C3, and F7 (Table 2).

Our neuropsychological assessments also indicated improvements in the posttreatment score as compared to baseline (Table 3).

Figure 1. Surface Maps of the Z-score Distribution (Full EEG).

Delta
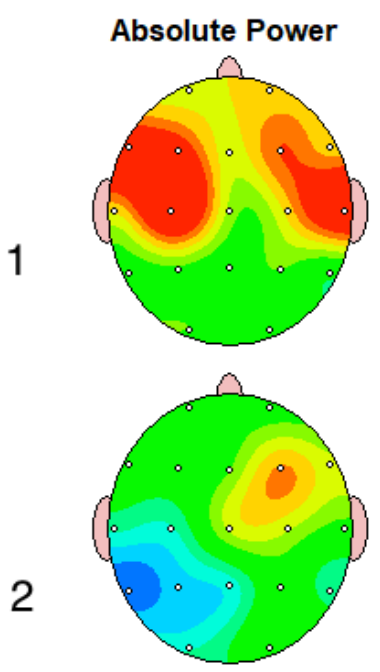

Theta
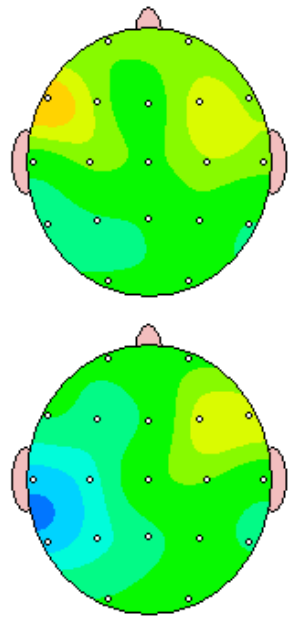

Alpha
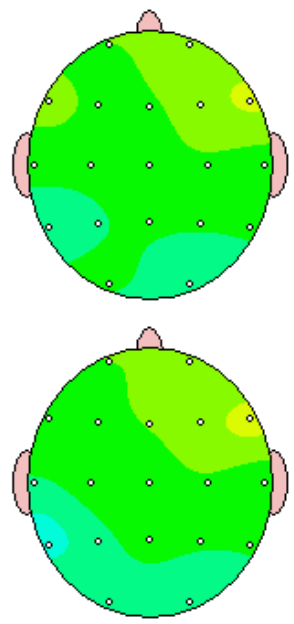

Beta
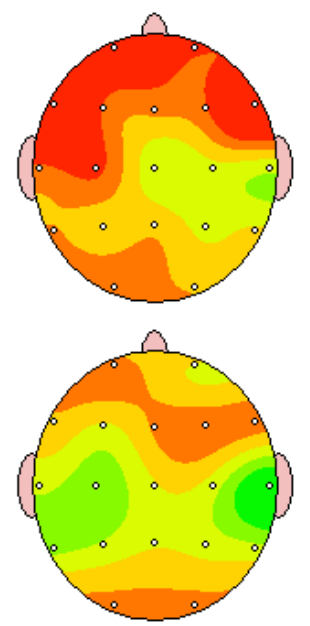

High Beta
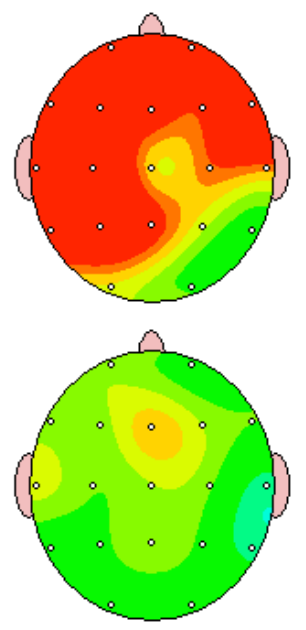

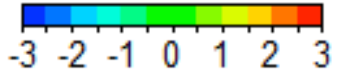

Note. The qEEG map shows the magnitude of deviations from the normal database using colors. The $z$-score $=0$ is defined as normal (green color). Scores less or more than the normal database are displayed by blue and red colors, respectively. EEG: Electroencephalography; 1 = Baseline qEEG; 2 = After 15 LZNFB sessions qEEG.

\section{Table 2}

The Largest Differences Between Baseline and Posttreatment

$\begin{array}{ccccccc}\text { Location } & \text { F8 } & \text { T4 } & \text { F7 } & \text { T5 } & \text { C3 } & \text { F7 } \\ \text { Brain wave } & \text { HB } & \text { HB } & \text { delta } & \text { HB } & \text { HB } & \text { HB } \\ \text { Percentage } & 89 \% & 88 \% & 86 \% & 84 \% & 84 \% & 81 \%\end{array}$

Note. $\mathrm{F}=$ Frontal; $\mathrm{T}=$ Temporal; $\mathrm{C}=$ Central; $\mathrm{HB}=$ High beta.

\section{Table 3}

Neuropsychological Test Scores Before and After LZNFB

\begin{tabular}{|c|c|c|c|c|}
\hline \multirow[t]{5}{*}{ Language test } & Language & Pretreatment & Posttreatment & $\Delta \mathrm{A}(\%)$ \\
\hline & Speed of speech & 32.9 & 53.7 & $38.7 \%$ \\
\hline & Lexical richness & 0.79 & 0.96 & $17.7 \%$ \\
\hline & Fluency & 6 & 7 & $14.2 \%$ \\
\hline & Total word number & 39 & 52 & $25 \%$ \\
\hline
\end{tabular}




\begin{tabular}{|c|c|c|c|c|}
\hline \multicolumn{5}{|c|}{$\begin{array}{l}\text { Table } 3 \\
\text { Neuropsychological Test Scores Before and After LZNFB }\end{array}$} \\
\hline \multirow[t]{3}{*}{ Working memory test } & Digit span & 6 & 10 & $40 \%$ \\
\hline & Word span & 6 & 8 & $25 \%$ \\
\hline & Nonword span & 4 & 4 & 0 \\
\hline \multirow[t]{2}{*}{ Stroop test } & $\begin{array}{l}\text { Correct answers } \\
\text { (congruent) }\end{array}$ & 28 & 48 & $39 \%$ \\
\hline & $\begin{array}{l}\text { Correct answer } \\
\text { (incongruent) }\end{array}$ & 21 & 46 & $54 \%$ \\
\hline
\end{tabular}

Note. LZNFB: LORETA Z-score neurofeedback. Clinically significant differences are shown in red $(\Delta A \%>15 \%$ is clinically significant).

\section{Discussion}

This study aimed to analyze the efficacy of LZNFB intervention for the treatment of aphasia following TBI. A qEEG-guided LZNFB protocol was designed for this purpose. Previous studies of TBI rehabilitation by NF have not focused on language performance. This study specifically evaluated the efficacy of LZNFB to rehabilitate the language deficit in a TBI patient. To this end, changes in qEEG/LORETA and aphasia battery metrics after 15 sessions of LZNFB were analyzed, as were changes in working memory and attention scores from pre- to posttreatment. The results showed that fifteen 40min NF sessions brought the EEG metrics within normal ranges and were effective in improving aphasia symptoms and cognitive performance. The findings of the current case study can be regarded as a promising addition to the treatment planning for TBI-related language problems in the future.

Our findings are consistent with those of previous studies regarding the effectiveness of NF on mitigating TBI symptoms (Bennett et al., 2018; Gray, 2017; Gupta et al., 2020; Kaser, 2020; Rostami et al., 2017).

\section{Effectiveness of LZNFB on the Electrophysiological Outcome}

At baseline qEEG demonstrated increased delta, theta, and beta bands at frontal and temporal locations, as well as decreased alpha at the posterior area. Increased delta and decreased alpha bands are known to be directly correlated with cortical metabolism (Szelies et al., 1999). The decreased alpha band at the posterior region and increased theta found in our study have also been seen in other studies (Arciniegas, 2011; Lewine et al., 2019). The increased delta and theta in our study are in line with those of the study of Moeller et al. (2011) and might be due to the disruption of the cortical-thalamic network in TBI. While increased beta occurred in this instance of TBI, it was not found in some similar studies (Leon-Carrion et al., 2008; Tebano et al., 1988). However, some other studies also found increased beta in TBI subjects (Randolph \& Miller, 1988; Thornton, 2003), with the researchers concluding that the increased beta was consistently a negative predictor of cognitive performance.

After 20 LZNFB sessions, the qEEG map showed an overall improvement (Figure 1). Our finding of neurological recovery by LZNFB is supported by previous studies that have confirmed its effectiveness in areas such as cerebrovascular accident rehabilitation (Koberda \& StodolskaKoberda, 2014), depression/anxiety and cognitive dysfunction (Koberda, 2015b), addiction (Faridi et al., 2020), attention-deficit/hyperactivity disorder (Koberda et al., 2014), pain management (Koberda et al., 2013), seizure (Koberda \& Frey, 2015), and TBI (Koberda, 2015a).

Based on the QEEG analysis, the largest differences between baseline and posttreatment were associated with the F8, F7, T5, and C3 locations (Table 2). The F8 and F7 electrodes correspond to BA 47, which is part of Broca's area and associated with the processing of syntax in oral and sign languages, musical syntax, and semantic aspects of language (Ardila, 2014). The T4 and T5 electrodes correspond to BA 22, which is located at the superior temporal gyrus and is part of Wernicke's area which is involved in speech comprehension. Further, the C3 electrode corresponds to BA 2, which is located in the primary somatosensory cortex, and the main function of this area is the cognitive control of language (Mofrad et al., 2020).

\section{Effectiveness of LZNFB on the Clinical Outcome}

Our assessment of the aphasia battery showed that P.F. had clinically significant recovery following treatment with LZNFB (Table 3). The clinical recovery of working memory and attention were also evident (Table 3). Several studies have reported the 
relationship between language and working memory (Emmorey et al., 2017; Fitz et al., 2020), as well as language and attention (Galassi et al., 2020; Peach et al., 2017; Vig et al., 2020; Villard \& Kiran, 2017; Wang et al., 2019), probably indicating that language is not independent of other cognitive performances; in other words, there is mutual interaction in this regard.

\section{Limitations}

This study had some limitations, including the sample size, which was a single case without a control group. Future studies would benefit from a larger sample size to maximize the power and accuracy of their results. In addition, exploring the relationship between TBI severity and LZNFB training effects may be a beneficial focus in the future.

\section{Conclusion}

The present preliminary findings suggest that LZNFB may have the potential to aid language performance among those with TBI. It was also found that the rehabilitation of the language network may improve working memory and attention in TBI cases. The result of this case highlights the need for investigating the efficacy of LZNFB not only as a treatment for aphasia but also as a tool for improving cognitive performance more generally.

\section{Author Declaration}

The authors declare that they have no grants, financial interests, or conflicts of interest to disclose.

\section{References}

Arciniegas, D. B. (2011). Clinical electrophysiologic assessments and mild traumatic brain injury: State-of-the-science and implications for clinical practice. International Journal of Psychophysiology, 82(1), 41-52. https://doi.org/10.1016 /j.ijpsycho.2011.03.004

Ardila, A. (2014). Aphasia handbook. Miami, FL: Florida International University.

Ayers, M. E. (1989, June). Electroencephalographic neurofeedback and closed head-injury of 250 individuals. Biofeedback and Self-regulation, 14(2), 130.

Banks, M. E. (2007). Overlooked but critical: Traumatic brain injury as a consequence of interpersonal violence. Trauma, Violence, \& Abuse, 8(3), 290-298. https://doi.org/10.1177 $/ 1524838007303503$

Barlow, D. Andrasik, F., \& Hersen, M. (2007). Single Case Experimental Designs: Strategies for Studying Behavior Change. London, UK: Allyn \& Bacon.

Barth, J. T., Macciocchi, S. N., Giordani, B., Rimel, R., Jane, J. A., \& Boll, T. J. (1983). Neuropsychological sequelae of minor head injury. Neurosurgery, 13(5), 529-533. https://doi.org /10.1227/00006123-198311000-00008

Bennett, C. N., Gupta, R. K., Prabhakar, P., Christopher, R., Sampath, S., Thennarasu, K., \& Rajeswaran, J. (2018). Clinical and biochemical outcomes following EEG neurofeedback training in traumatic brain injury in the context of spontaneous recovery. Clinical EEG and Neuroscience, 49(6), 433-440. https://doi.org/10.1177/1550059417744899

Brigo, F., \& Mecarelli, O. (2019). Traumatic brain injury. In O. Mecarelli (Ed.), Clinical electroencephalography (pp. 617622). Switzerland: Springer International Publishing.

Brown, J., Clark, D., \& Pooley, A. E. (2019). Exploring the use of neurofeedback therapy in mitigating symptoms of traumatic brain injury in survivors of intimate partner violence. Journal of Aggression, Maltreatment \& Trauma, 28(6), 764-783. https://doi.org/10.1080/10926771.2019.1603176

Emmorey, K., Giezen, M. R., Petrich, J. A. F., Spurgeon, E., \& Farnady, L. O. G. (2017). The relation between working memory and language comprehension in signers and speakers. Acta Psychologica, 177, 69-77. https://doi.org /10.1016/j.actpsy.2017.04.014

Faridi, A., Taremian, F., Thatcher, R. W., Dadashi, M., \& Moloodi, R. (2020). Comparison of LORETA $z$ score neurofeedback and cognitive rehabilitation in terms of their effectiveness in reducing craving in opioid addicts [Manuscript accepted for publication]. Basic and Clinical Neuroscience. https://doi.org /10.32598/bcn.2021.1946.1

Fitz, H., Uhlmann, M., Van den Broek, D., Duarte, R., Hagoort, P., \& Petersson, K. M. (2020). Neuronal spike-rate adaptation supports working memory in language processing. Proceedings of the National Academy of Sciences of the Unites States of America, 117(34), 20881-20889. https://doi.org/10.1073/pnas.2000222117

Galassi, A., Lippi, M., \& Torroni, P. (2020). Attention in natural language processing. IEEE Transactions on Neural Networks and Learning Systems, 1-18. http://dx.doi.org/10.1109 /TNNLS.2020.3019893

Galovic, M., Schmitz, B., \& Tettenbom, B. (2017). EEG in inflammatory disorders, cerebrovascular diseases, trauma and migraine. In D. L. Schomer, \& F. H. L. da Silva (Eds.), Niedermeyer's electroencephalography: Basic principles, clinical applications, and related fields (7th ed., pp. 371-412). Oxford, UK: Oxford University Press. https://doi.org/10.1093 $/ \mathrm{med} / 9780190228484.003 .0015$

Gray, S. N. (2017). An overview of the use of neurofeedback biofeedback for the treatment of symptoms of traumatic brain injury in military and civilian populations. Medical Acupuncture, 29(4), 215-219. https://doi.org/10.1089 /acu.2017.1220

Gupta, R. K., Afsar, M., Yadav, R. K., Shukla, D. P., \& Rajeswaran, J. (2020). Effect of EEG neurofeedback training in patients with moderate-severe traumatic brain injury: $A$ clinical and electrophysiological outcome study. NeuroRegulation, 7(2), 75-83. https://doi.org/10.15540 /nr.7.2.75

Hershaw, J. N., Hill-Pearson, C. A., Arango, J. I., Souvignier, A. R., \& Pazdan, R. M. (2020). Semi-automated neurofeedback therapy for persistent postconcussive symptoms in a military clinical setting: A feasibility study. Military Medicine, 185(3-4), e457-e465. https://doi.org/10.1093/milmed/usz335

Jackson, H., Philp, E., Nuttall, R. L., \& Diller, L. (2002). Traumatic brain injury: A hidden consequence for battered women. Professional Psychology: Research and Practice, 33(1), 3945. https://doi.org/10.1037/0735-7028.33.1.39

Kaser, A. (2020). The influence of neurofeedback training on verbal episodic memory in a TBI sample. Waco, TX: Baylor University.

Koberda, J. L. (2015a). LORETA Z-score neurofeedbackeffectiveness in rehabilitation of patients suffering from traumatic brain injury. Journal of Neurology and Neurobiology, 1(4), 1-9. https://doi.org/10.16966/23797150.113

Koberda, J. L. (2015b). Z-score LORETA neurofeedback as a potential therapy in depression/anxiety and cognitive dysfunction. In R. W. Thatcher \& J. F. Lubar (Eds.), Z score 
neurofeedback: Clinical applications (pp. 93-113). San Diego, CA: Academic Press.

Koberda, J., \& Frey, L. (2015). Z-score LORETA neurofeedback as a potential therapy for patients with seizures and refractory epilepsy. Journal of Neurology and Neurobiology, 1(1). https://doi.org/10.16966/2379-7150.101

Koberda, J. L., Koberda, P., Bienkiewicz, A. A., Moses, A., \& Koberda, L. (2013). Pain management using 19-electrode Zscore LORETA neurofeedback. Journal of Neurotherapy, 17(3), 179-190. https://doi.org/10.1080 /10874208.2013.813204

Koberda, J. L., Koberda, P., Moses, A., Winslow, J., Bienkiewicz, A., \& Koberda, L. (2014). Z-score LORETA neurofeedback as a potential therapy for ADHD. Biofeedback, 42(2), 74-81. https://doi.org/10.5298/1081-5937-42.2.05

Koberda, J. L., \& Stodolska-Koberda, U. (2014). Z-score LORETA neurofeedback as a potential rehabilitation modality in patients with CVA. Journal of Neurology \& Stroke, 1(5), 00029. https://doi.org/10.15406/jnsk.2014.01.00029

Leon-Carrion, J., Martin-Rodriguez, J. F., Damas-Lopez, J., Y Martin, J. M. B., \& Dominguez-Morales, M. D. R. (2008). A QEEG index of level of functional dependence for people sustaining acquired brain injury: The Seville Independence Index (SINDI). Brain Injury, 22(1), 61-74. https://doi.org $/ 10.1080 / 02699050701824143$

Lewine, J. D., Plis, S., Ulloa, A., Williams, C., Spitz, M., Foley, J., Paulson, K., Davis, J., Bangera, N., Snyder, T., \& Weaver, L. (2019). Quantitative EEG biomarkers for mild traumatic brain injury. Journal of Clinical Neurophysiology, 36(4), 298-305. https://doi.org/10.1097/WNP.0000000000000588

Modarres, M. H., Kuzma, N. N., Kretzmer, T., Pack, A. I., \& Lim, M. M. (2017). EEG slow waves in traumatic brain injury: Convergent findings in mouse and man. Neurobiology of Sleep and Circadian Rhythms, 2, 59-70. https://doi.org /10.1016/j.nbscr.2016.06.001

Moeller, J. J., Tu, B., \& Bazil, C. W. (2011). Quantitative and qualitative analysis of ambulatory electroencephalography during mild traumatic brain injury. Archives of Neurology, 68(12), 1595-1598. $\quad$ https://doi.org/10.1001 /archneurol.2011.1080

Mofrad, F. T., Jahn, A., \& Schiller, N. O. (2020). Dual function of primary somatosensory cortex in cognitive control of language: Evidence from resting state fMRI. Neuroscience, 446, 59-68. https://doi.org/10.1016 /j.neuroscience.2020.08.032

Peach, R. K., Nathan, M. R., \& Beck, K. M. (2017). Languagespecific attention treatment for aphasia: Description and preliminary findings. Seminars in Speech and Language, 38(1), 5-16. https://doi.org/10.1055/s-0036-1597260

Randolph, C., \& Miller, M. H. (1988). EEG and cognitive performance following closed head injury. Neuropsychobiology, 20(1), 43-50. https://doi.org/10.1159 1000118471

Rostami, R., Salamati, P., Yarandi, K. K., Khoshnevisan, A., Saadat, S., Kamali, Z. S., Ghiasi, S., Zaryabi, A., Ghazi Mir Saeid, S. S., Arjipour, M., Rezaee-Zavareh, M. S., \& RahimiMovaghar, V. (2017). Effects of neurofeedback on the shortterm memory and continuous attention of patients with moderate traumatic brain injury: A preliminary randomized controlled clinical trial. Chinese Journal of Traumatology, 20(5), 278-282. https://doi.org/10.1016/j.cjtee.2016.11.007

Szelies, B., Mielke, R., Kessler, J., \& Heiss, W.-D. (1999). EEG power changes are related to regional cerebral glucose metabolism in vascular dementia. Clinical Neurophysiology, 110(4), 615-620. https://doi.org/10.1016/S13882457(98)00052-2

Tebano, M. T., Cameroni, M., Gallozzi, G., Loizzo, A., Palazzino, G., Pezzini, G., \& Ricci, G. F. (1988). EEG spectral analysis after minor head injury in man. Electroencephalography and Clinical Neurophysiology, 70(2), 185-189. https://doi.org /10.1016/0013-4694(88)90118-6

Thatcher, R. W. (2010). LORETA Z score biofeedback. NeuroConnections, 9-13.

Thornton, K. (2003). The electrophysiological effects of a brain injury on auditory memory functioning: The QEEG correlates of impaired memory. Archives of Clinical Neuropsychology, 18(4), 363-378. https://doi.org/10.1093/arclin/18.4.363

Vig, J., Madani, A., Varshney, L. R., Xiong, C., Socher, R., \& Rajani, N. F. (2020). BERTology meets biology: Interpreting attention in protein language models [preprint]. ICLR 2021, arXiv:2006.15222 [cs.CL].

Villard, S., \& Kiran, S. (2017). To what extent does attention underlie language in aphasia? Aphasiology, 31(10), 12261245. https://doi.org/10.1080/02687038.2016.1242711

Wang, P., Wu, Q., Cao, J., Shen, C., Gao, L., \& van den Hengel, A. (2019). Neighbourhood watch: Referring expression comprehension via language-guided graph attention networks. Proceedings of the IEEE Conference on Computer Vision and Pattern Recognition (CVPR), 2019, 1960-1968.

Received: January 21, 2021

Accepted: April 24, 2021

Published: June 30, 2021 